Bridging the Gaps in Palliative Care Bereavement Support: An International

\title{
Perspective
}

\author{
Lauren J. Breen ${ }^{1}$ \\ Samar M. Aoun ${ }^{2}$ \\ Moira O’Connor ${ }^{1}$ \\ Bruce Rumbold ${ }^{3}$
}

${ }^{1}$ School of Psychology and Speech Pathology, Faculty of Health Sciences, Curtin

University, Perth, Australia

${ }^{2}$ School of Nursing and Midwifery, Faculty of Health Sciences, Curtin University, Perth, Australia

${ }^{3}$ School of Public Health, LaTrobe University, Melbourne, Australia

Keywords: Bereavement; Caregiving; Counselling; End-of-life; Palliative care; Public health approach

Acknowledgments: The first author is supported by the Australian Research Council (DE120101640) and a PsyLife Research Grant. 


\begin{abstract}
A review of palliative care policies and bereavement support practices in the United States, Canada, United Kingdom, Australia, and Japan demonstrated four challenges questions over providing universal versus targeted support; a lack of clear evidence driving service delivery; informal or no risk assessment; and limited or no evaluation of services. Bridging the gaps between the policy and practice of palliative care bereavement support requires acknowledging the challenges of medical and public health models, improving bereavement need assessment processes, enhancing the role of primary care providers, and extending research to provide an evidence-base for interventions.
\end{abstract}

Keywords: Bereavement; Caregiving; Counselling; End-of-life; Palliative care; Public health approach 


\section{Bridging the Gaps in Palliative Care Bereavement Support: An International Perspective}

Palliative care is holistic support for people living with terminal illness. A central tenet of palliative care is support for patients and family caregivers throughout the illness and for these caregivers following bereavement (Sepv́lveda, Marlin, Yoshida, \& Ullrich, 2002). However, palliative care services grapple with how best to use their resources for bereavement care. Although the World Health Organization (2007) describes palliative care as using "a team approach to address the needs of patients and their families, including bereavement counselling, if indicated" (p. 3, emphasis added), most palliative care services tend to provide blanket bereavement interventions to all carers and families regardless of their support needs.

In this paper, we address the ways industrialised societies support bereaved people by examining policies, practices, and identified gaps in bereavement services in palliative care. We begin by summarising the current state of bereavement interventions in palliative care in the United States, United Kingdom, Canada, Australia, and Japan. Next, we provide an overview of who needs what kind of bereavement interventions, when they will most benefit from them, and how the different needs of bereaved people may be distinguished. Finally, we propose a way forward for the application of a public health approach to bereavement support in palliative care in order to bridge the gaps between policy and practice.

\section{Bereavement Services in Palliative Care: An International Analysis}

Despite the increasing need for palliative care, especially in industrialised countries, and bereavement support being a core component of palliative care, formal services and supports available to patients' caregivers and families post-bereavement vary significantly between countries (O’Connor, Abbott, Payne, \& Demmer, 2009). An 
analysis of bereavement care practices in five countries with established palliative care services demonstrates that these services struggle to use their limited resources to deliver best practice bereavement care and support. These countries were chosen because we could access policy (except Japan) and research on practice in English.

\section{United States}

In the United States, the National Hospice and Palliative Care Organization (2008) developed practice standards for bereavement care. These standards stress the importance of identifying family members in need of bereavement support, assessing bereavement risk, and referring clients with complicated bereavement to other services. A survey of 131 hospices in California demonstrated that the most common types of support were telephone calls (offered by $98 \%$ of services), mailed information (96\%), grief brochures (95\%), pastoral care (90\%), visits by volunteers (82\%), professional counselling (58\%), support groups (50\%), and workshops (47\%); the vast majority of hospices (89\%) provided these services free of charge (Foliart, Clausen, \& Siljestrom, 2001). Almost onequarter of personnel providing bereavement support were trained volunteers and $11 \%$ of staff involved closely in bereavement support had no formal qualification. These services were available to every bereaved family, irrespective of need.

Similarly, 260 hospice and palliative care services surveyed in the United States revealed that they aimed to provide bereavement services to all bereaved individuals (Demmer, 2003). Most (92\%) reported assessment to determine individuals at-risk; however, $87 \%$ used an in-house assessment tool rather than a standardised measure. Only one-third employed a full-time bereavement coordinator and this role was part-time in two-thirds of these services. The most common supports offered were letters/notices of groups and meetings (87\% of services), telephone calls (83\%), and information on grief (81\%). Just over half of the services $(55 \%)$ reported engaging in home visits. Other 
specific services included memorial services (46\%), newsletters (38\%), children's camps (27\%), and support groups (23\%). Obstacles to service delivery were limited staff time, funding pressures, lack of client interest, and lack of organisational support for bereavement services.

\section{Canada}

The Canadian Hospice Palliative Care Association proposed a national model for hospice and palliative care wherein families receive bereavement care; however, it does not specify whether and how this care is delivered (Ferris et al., 2002). The provision of counselling, bereavement, and spiritual support accounts for $14 \%$ of the annual operating budget of Canada's Victoria Hospice (Victoria Hospice, 2010). In Alberta, families receive a bereavement package consisting of grief information and community supports and resources; however, data are not available for the 2008-2009 year due to the Grief Manager position being vacant (Alberta Health Services, 2009).

\section{United Kingdom}

The National Institute for Health and Clinical Excellence (NICE, 2004) in the United Kingdom proposed an approach to bereavement in palliative care whereby caregivers and families receive support according to their needs. These guidelines recommend the assessment of family carers to determine their support needs with support provided accordingly. A study published the same year as the NICE guidelines showed that bereavement care was most commonly offered on a universal basis, irrespective of need. For instance, of 248 palliative care and hospice services surveyed in the United Kingdom, fewer than half (43\%) used a formal assessment tool to determine need for bereavement services (Field, Reid, Payne, \& Relf, 2004). The most common services offered were one-to-one counselling (offered by $96 \%$ of services), telephone support (90\%), referral (89\%), memorial/remembrance services (72\%), written information (73\%), 
and support groups (61\%). Service delivery was obfuscated by a need for more staff and limited coordination of bereavement services.

An in-depth analysis of five United Kingdom hospices showed that, although based upon different philosophies of care, they tended to provide similar types of bereavement supports - information on bereavement, telephone or written contact with the bereaved, and psychosocial support from volunteer befrienders and/or paid counsellors (Reid, Field, Payne, \& Relf, 2006a). Furthermore, although no service used a formal method of risk assessment following bereavement, all five engaged in a process of offering support to all of the bereaved that allowed them to 'opt-in' to receive these supports. These authors concluded that, by focussing limited resources on blanket service delivery, these services were unequipped to support people with more complex bereavement needs (Reid et al., 2006b). This universal provision of bereavement services in palliative care has been criticised for ignoring the NICE guidelines:

The 'universalistic' approach of offering bereavement support to all eligible adult reported in this paper is consistent with the general philosophy of UK hospices to care for patient and their family. However, it is inconsistent with NICE guidelines to use 'risk assessment' to identify those people most likely to need support. (Reid et al., 2006a, p. 327)

A more recent analysis of 10 bereavement services in palliative care in the United Kingdom found that all 10 services used formal assessment processes, typically a nonstandardised bereavement checklist based on one or more theoretical bereavement models, to identify the needs of bereaved family members (Agnew, Mankletow, Haynes, \& Jones, 2011). However, 7 of the 10 services expressed dissatisfaction with the assessment processes for being incomplete, inaccurate or ambiguous, and for not being evidencebased. 
Australia

Palliative Care Australia's Standard 8 asserts, "Formal mechanisms are in place to ensure that the patient, their caregiver/s and family have access to bereavement care, information and support services" (Palliative Care Australia, 2005, p. 33) and adds that this support should be based on need. Recent clinical practice guidelines for bereavement supports in palliative care also highlight the importance of providing support according to the assessment of need (Hudson et al., 2010). A recent audit of Australian palliative care services established bereavement care as the highest priority for improvement (Palliative Care Australia, 2010); this report, along with the latest national palliative care strategy (Commonwealth of Australia, 2010), recommended a national evidence-based approach to bereavement support.

However, recent surveys demonstrate that almost all Australian palliative care services offer bereavement supports and services to families of deceased patients, regardless of risk or need. For instance, one survey of 236 Australian palliative care services determined that $95 \%$ offered some form of bereavement support (Mather, Good, Cavenagh, \& Ravenscroft, 2008). The authors of this study concluded that there is "lack of clear evidence to guide development and allocation of bereavement programs in palliative care” (p. 230).The most common types of support were a telephone call (offered by 86\%), memorial service, (66\%), letter (55\%), anniversary card (53\%), group sessions (31\%), information package (5\%), and informal gatherings (4\%). Approximately two-thirds of the services reported engaging in some form of bereavement risk assessment, with two-thirds relying on multidisciplinary team opinion, more than half using a formal tool to assess bereavement risk, and approximately half relying on a single staff member's opinion (some services reported using more than one method). 
Another survey of 143 Australian palliative care and hospice services showed that 94\% provide universal bereavement services, with only three services limiting bereavement services solely to high-risk caregivers (Abbott, O’Connor, \& Payne, 2008). Two-thirds reported using some type of bereavement risk assessment, typically an informal assessment form, in order to determine risk and fewer than $10 \%$ used a formal assessment tool. Among these services, the ability to meet clients' needs with the services' resources was a significant concern, with Abbott et al. (2008) concluding: ...our data suggest that many services struggle to provide what they would wish, because of the models of bereavement support used, the lack [of] formal assessment of risk, and personnel and funding contracts. Questions remain about the value of providing bereavement support to all, rather than allocating services based upon level of need. (p. 17)

The services highlighted a lack of staff time, funding pressures, and limited organisational support for bereavement services as primary obstacles to service delivery (Abbott et al., 2008).

Japan

Hospice and palliative care services are recent developments in Japan; however, the country appears to be taking its lead from English-speaking countries. Two surveys of hospices and palliative care services revealed that the majority of services (74-95\%) provided some bereavement follow-up (Matsushima, Akabayashi, \& Nishitateno, 2002; Sakaguchi et al., 2004). Strategies documented in the two studies included memorial cards (84-89\%), memorial services (59-75\%), social group meetings (35\%), telephone calls (32$64 \%$ ), attending funerals (39\%), providing information (38\%), individual counselling (22$40 \%$ ), home visits (22-27\%), mutual-help groups (11\%), family counselling (8-31\%), and group counselling (3-19\%). A range of staff members including nurses, social workers, 
religious workers, physicians, volunteers, and counsellors provided these services (Matsushima et al., 2002). Challenges included inadequate staff training and resources, limited organisational support for bereavement care in hospice and palliative care services, and the desire to match support to need (Matsushima et al., 2002). In particular, nearly half $(46 \%)$ of the services preferred for supports to be provided by a bereavement coordinator, although none was employed (Sakaguchi et al., 2004).

Overall, a review of bereavement support in palliative care in five countries demonstrates four respects in which practice is inadequate - questions over providing universal versus targeted support; a lack of clear evidence driving service delivery; informal or no risk assessment; and limited to no evaluation of services. Palliative care services grapple with issues such as who should be offered bereavement interventions, when and how long these interventions should be offered, and how the efficacy of interventions can be maximised given funding and staffing constraints.

Bereavement Needs and Interventions: Who Needs What and When?

Not all bereaved people suffer a significant and long-term grief reaction following the death of a loved one (Bonanno, Boerner, \& Wortman, 2008); however, some bereaved caregivers do exhibit elevated levels of distress following the death of their loved one. Although most caregivers experience short-lived distress following bereavement, a significant minority (about 10-20\%) experience persistent psychiatric difficulties (Prigerson, Vanderwerker, \& Maciejwski, 2008) and would benefit from professional intervention.

Research on complications of bereavement is growing rapidly. Prolonged Grief Disorder (formerly known as traumatic grief and complicated grief disorder) is under review for inclusion in the World Health Organization's International Classification of Diseases (ICD-11; Maerker et al., in press) and Persistent Complex Bereavement Disorder 
was included as a category for further study in the latest Diagnostic and Statistical Manual of Mental Disorders (DSM-5; American Psychiatric Association, 2013). Several factors contribute to the risk of complicated grief and include factors prior to bereavement such as previous loss, psychiatric history, relationship with the deceased, attachment style, and exposure to trauma, and factors at the time of death such as mode of death, death preparedness, and lack of social support (Lobb et al., 2010).

Grief interventions show differential efficacy according to level of need. A comprehensive meta-analysis of 61 controlled studies of psychotherapeutic interventions for bereavement (Currier, Neimeyer, \& Berman, 2008) demonstrated greater efficacy for interventions targeted to grievers with higher levels of distress (e.g., clinical symptomatology). Grief interventions for individuals with 'normal' grief tend to offer minimal to no efficacy and may even result in greater distress (e.g., Schut \& Stroebe, 2005). The move to offering support according to need is strengthened by recent empirical studies that indicate support for targeted interventions for people who meet the criteria for Prolonged Grief Disorder (e.g., Boelen, de Keijser, van den Hout, van den Bout, 2007). Clearly, a blanket approach to bereavement care is not appropriate; instead, the focus needs to shift towards service delivery models predicated upon individual need.

\section{Models of Bereavement Interventions: Determining Who Gets What and When?}

Three levels of preventive intervention for bereavement care (Schut, Stroebe, van den Bout, \& Terheggen, 2001) are: primary - targeting all bereaved people; secondary targeting people at-risk of complications of bereavement; and tertiary - targeting people with complicated bereavement. These levels of intervention align with Neimeyer's (2008) differentiation of grief support (informal compassion and information from people who do not have professional bereavement training), grief counselling (provided by trained 
professionals), and grief therapy (provided by trained professionals to people with mental health concerns).

The United Kingdom's NICE (2004) proposed a three-tiered approach to bereavement in palliative care according to the needs of caregivers and families. Palliative care services should universally provide information about bereavement and appropriate supports, with much of the support coming from the bereaved person's social networks, including compassionate family members and friends (Component 1). A smaller proportion would benefit from non-specialist social and therapeutic support from sources such as volunteer bereavement workers, bereavement mutual-help groups, and faith-based and other community groups (Component 2). An even smaller proportion would gain from specialist intervention such as counselling, mental health services, bereavement services, or psychotherapy (Component 3). Providing high-quality bereavement support for those with complex needs and those at-risk of complex needs may prevent further pathology (Prigerson et al., 2001) and significantly reduce use of health services, particularly visits to general practitioners (Vanderwerker, Laff, Kadan-Lottick, McColl, \& Prigerson, 2005). We (Aoun, Breen, O’Connor, Rumbold, \& Nordstrom, 2012) advocate a public health approach to bereavement care to encompass the NICE (2004) guidelines within a partnership model between the health sector and the community. Local communities have a key role in supporting people living with chronic illness nearing end-of-life (Salau, Rumbold, \& Young, 2007). Underlying this public health approach is the notion that death, dying, and grief are normal aspects of human experience, and that communities need some resilience around end-of-life events to live together with loss. Community supports provide an ideal mechanism for the provision of components 1 and 2. Social support is important for adaptation to, and recovery from, bereavement and most of this comes from natural sources of social support - family and friends (Benkel, Wijk, \& 
Molander, 2005). Nevertheless, not everyone has adequate social support networks and, in some cases, particularly in stigmatising and disenfranchised bereavement, there may be a breakdown of these networks (Breen \& O'Connor, 2011). One study of family members of patients who had received palliative care found that $70 \%$ would have liked support following bereavement. These respondents preferred receiving this support from people who had had a similar experience rather than a specialised professional (Jansma, Schure, \& deJong, 2005).

\section{Bridging the Gaps between Policy and Practice}

Despite rising interest in providing bereavement care in palliative care, there is a clear misalignment between palliative care policy and actual practice. This incongruence between research and practice remains a critically important issue in the bereavement literature (Breen \& O’Connor, 2007; Neimeyer, Harris, Winokuer, \& Thornton, 2011). The disparity is also problematic in that offering professional support to all bereaved people is neither effective nor economical. Health-funding bodies have limited pools of money to spend on bereavement care so balancing need with resources is of utmost importance. For example, less than $5 \%$ of the palliative care budget in the United States and Australia is directed to bereavement services (O’Connor et al., 2009). In proposing a public health approach to bridge the gaps between the policy and practice of palliative care bereavement support, models of health and helping require consideration.

First, the medical model influences bereavement care. This model views people requiring services as passive, the focus is on an individual's functional limitations or psychological losses, the aim is to administer 'care' or a 'cure' and the health professional is the 'expert' who intervenes to provide the solution (Brickman et al., 1982). Accordingly, health services focus on abnormality and, as a result, most strategies for bereavement focus on the small proportion of people who struggle rather than the large 
majority who do not. This "production of bereavement care" (Small \& Hockey, 2001, p. 97), is characterised by the emergence of bereavement counselling as "an industry and an institution" (Árnason, 2001a, p. 299), and is essential in the management of death and grief (Árnason, 2001b), has occurred in tandem with the subordination of lay understandings of grief and the invisibility of death and dying unless a medical 'problem' is identified.

Second, public health approaches tend to focus on extending existing health services to whole populations, yet Aoun et al. (2012) articulate that blanket bereavement support for everyone, underpinned by this one-size-fits-all approach, is not effective or economical. Instead, matching client need to care received is paramount. A public health approach to bereavement services in palliative care provides an innovative way of differentiating bereavement that would benefit from palliative care services from bereavement that might be better managed elsewhere. Applied to palliative care, it provides a systematic and evidence-based way of meeting the needs of bereaved family caregivers while reducing economic and staffing constraints. Appropriate screening should direct some people to the resources they need while keeping others from harm, but it is the perspective of screening that matters. The challenges we emphasise have arisen because surveillance of bereavement occurred first in medical terms and then more recently in grief counselling terms (Conway, 2011). A possible consequence of the uncritical incorporation of bereavement screening into current approaches is the risk that such screening will become another avenue for 'health moralism' emerging from some health promotion efforts (see Alcabes, 2003). Bearing in mind these risks, we need to develop solutions for moving forward with a public health approach.

Evidence-based practice is a necessity in healthcare, including palliative care, but, so far, has limited reach beyond the patient. It is a logical progression that bereaved carers 
become the next focus, especially as palliative care continues towards increased professionalisation. Identifying the proportions of bereaved people in need of the three components of support (information and compassion, non-specialised support, and specialist intervention) within each jurisdiction will facilitate the development, offer, and evaluation of a range of community-based programs to meet the varied needs of bereaved people. In achieving the latter, there is scope to use community-based, participatory research methods to work in partnership with palliative care services (Hopkinson, Wright, \& Corner, 2005) to produce feasible bereavement care assessment and referral pathways and the Reach Effectiveness Adoption Implementation Maintenance (RE-AIM) framework to evaluate the implementation of such innovation (Glasgow, Vogt, \& Boles, 1999).

There is currently an over-reliance on risk assessment tools that are not reliably predictive. Hansson and Stroebe (2007) propose an integrative risk factor framework that takes into account bereavement factors (e.g., relationship to deceased, model of death), interpersonal factors (e.g., social support, family dynamics), intrapersonal risk factors (e.g., personality, religious beliefs, existing vulnerability), and appraisal and coping styles, in predicting bereavement outcome. Developing and validating a tool incorporating these factors would be advantageous as current commonly-used bereavement risk assessment tools are criticised for their deficient theoretical bases, inadequate psychometrics, and limited applicability to palliative care (Agnew, Mankletow, Taylor, \& Jones, 2010).

Furthermore, a key limitation of our current understanding is that we know comparatively little about bereavement services following unexpected deaths. Even when deaths are expected, people with advanced cancer and other terminal illnesses are typically referred to palliative care very late in their illness trajectory or not referred at all (Haines, 2011). The consequence of this narrow focus is that we know surprisingly little about 
'normal' bereavement, the effectiveness of interventions other than counselling, how people and communities support each other, and how communities might further develop ways of supporting each other in, and preparing for, bereavement. In our attempts to bridge the gaps between policy and practice, a public health approach to bereavement support may be applied beyond palliative care settings, which represent a small, niche proportion of the entire bereaved population.

One essential community support intervention that would be underpinned by research is the development of an educational intervention for service providers in primary care. In one survey, general practitioners rated their knowledge in death, dying, and loss as low(O'Connor \& Lee-Steere, 2006) and, as such, there is a need for further education and professional development to enhance their knowledge in these areas. An additional source of community support hitherto untapped is community pharmacists. With education, community pharmacists are well-positioned to provide support in the community to patients and families before and after death (O’Connor, Pugh, Jiwa, Hughes, \& Fisher, 2011). Community pharmacists are willing to be involved in the palliative care team, but they report a need for professional development opportunities in communicating effectively with carers, particularly after bereavement (O'Connor et al., 2011).

\section{Conclusion}

A public health approach, then, must begin with the diversity of population needs, responses, and outcomes. It should take into account the need for professional psychological help in prolonged grief, ask about other professional healthcare interventions and support that might be appropriate for normal grief, and reflect on how to strengthen and enhance informal care and support in the community. It should also consider preventive care, especially strategies to promote the development of resilience in 
individuals and communities and reduce the incidence of prolonged grief, so that bereavement is not an isolating, catastrophic event but as an expected and ultimately survivable aspect of human experience. Developing such a public health approach requires that we complement our medical and psychological understandings, which are currently over-emphasised, with social, cultural, and spiritual strategies. We need to focus on the next steps in building resilience in the communities in which we belong, so that we can support each other without necessarily seeing end-of-life events as problems to be solved or situations merely to be endured. 


\section{References}

Abbott, J., O'Connor, M., \& Payne, S. (2008). An Australian survey of palliative care and hospice bereavement services. The Australian Journal of Cancer Nursing, 9(2), $12-$ 17.

Agnew, A., Manktelow, R., Haynes, T., \& Jones, L. (2011). Bereavement assessment practice in hospice settings: Challenges for palliative care social workers. British Journal of Social Work, 41, 111-130.

Agnew, A., Manktelow, R., Taylor, B. J., \& Jones, L. (2010). Bereavement needs assessment in specialist palliative care: A review of the literature. Palliative Medicine, 24, 46-59.

Alberta Health Services. (2009). Regional palliative care program-balanced scorecard report 2008-2009. Edmonton, Canada: Author. Accessed $19^{\text {th }}$ July 2011 from http://www.albertahealthservices.ca/default.asp

Alcabes, P. (2003, May 23). Epidemiologists need to shatter the myth of a risk-free life. The Chronicle. Accessed September 6, 2011, from http://www.ph.ucla.edu/epi/bioter/shattermyth.html

American Psychiatric Association. (2013). Diagnostic and statistical manual of manual disorders $\left(5^{\text {th }}\right.$ ed.). Washington, DC: Author.

Aoun, S., Breen, L. J., O’Connor, M., Rumbold, B., \& Nordstrom, C. (2012). A public health approach to bereavement support services in palliative care. Australian and New Zealand Journal of Public Health, 36, 14-16.

Árnason, A. (2001a). Experts of the ordinary: Bereavement counseling in Britain. Journal of the Royal Anthropological Institute, 7, 299-313. 
Árnason, A. (2001b). The skills we need: Bereavement counselling and governmentality in England. In J. Hockey, J. Katz, \& N. Small (Eds.), Grief, mourning and death ritual (pp. 125-134). Buckingham, UK: Open University Press.

Benkel, I., Wijk, H., \& Molander, U. (2005). Family and friends provide most social support for the bereaved. Palliative Medicine, 23, 141-149.

Boelen, P. A., de Keijser, J., van den Hout, M. A., \& van den Bout, J. (2007). Treatment of complicated grief: A comparison between cognitive-behavioural therapy and supportive counselling. Journal of Consulting and Clinical Psychology, 75, 277-284.

Bonanno, G. A., Boerner, K., \& Wortman, C. B. (2008). Trajectories of grieving. In M. S. Stroebe, R. O. Hansson, H. Schut, \& W. Stroebe (Eds.), Handbook of bereavement research and practice: Advances in theory and intervention (pp. 287-307).

Washington, DC: American Psychological Association.

Breen, L. J., \& O’Connor, M. (2007). The fundamental paradox in the grief literature: A critical reflection. Omega, 55, 199-218.

Breen, L. J., \& O'Connor, M. (2011). Family and social networks after bereavement: Experiences of support, change, and isolation. Journal of Family Therapy, 33, 98120.

Brickman, P., Rabinowitz, V. C., Karuza, J. Jr, Coates, D., Cohn, E., Kidder, L. (1982). Models of helping and coping. American Psychologist, 37, 368-384.

Commonwealth of Australia. (2010). National palliative care strategy. Barton, Australian Capital Territory, Australia: Author. Accessed 21 ${ }^{\text {st }}$ September 2011 from http://www.health.gov.au/internet/main/publishing.nsf/Content/palliativecarestrategy.htm

Conway, S. (Ed.). (2011). Governing death and loss: Empowerment, involvement and participation. Oxford, UK: Oxford University Press. 
Currier, J. M., Neimeyer, R. A., \& Berman, J. S. (2008). The effectiveness of psychotherapeutic interventions for bereaved persons: A comprehensive quantitative review. Psychological Bulletin, 134, 648-661.

Demmer, C. (2003). A national survey of hospice bereavement services. Omega, 47, 327 341.

Ferris, F., D., Balfour, H. M., Bowen, K., Farley, J., Hardwick, M., Mamontagne, C., ... \& West, P. (2002). A model to guide hospice palliative care: Based on national principles and norms of practice. Ottawa, Ontario, Canada: Canadian Hospice Palliative Care Association. Accessed $19^{\text {th }}$ July 2011 from http://www.chpca.net/model_to_guide_hpc

Field, D., Reid, D., Payne, S., \& Relf, M. (2004). Survey of UK hospice and specialist palliative care adult bereavement services. International Journal of Palliative Nursing, 10, 569-576.

Foliart, D. E., Clausen, M., \& Siljestrom, C. (2001). Bereavement practices among California hospices: What do palliative support teams actually do? Death Studies, 25, 461-467.

Glasgow, R. E., Vogt, T. M., \& Boles, S. M. (1999). Evaluating the public health impact of health promotion interventions: The RE-AIM framework. American Journal of Public Health, 89, 1322-1327.

Haines, I. E. (2011). Managing patients with advanced cancer: The benefits of early referral for palliative care. Medical Journal of Australia, 194, 107-108.

Hansson, R. O., \& Stroebe, M. S. (2007). Bereavement in late life: Coping, adaptation, and developmental influences. Washington, DC: American Psychological Association. 
Hopkinson, J. B., Wright, D. N. M., \& Corner, J. L. (2005).Seeking new methodology for palliative care research: Challenging assumptions about studying people who are approaching the end of life. Palliative Medicine, 19, 532-537.

Hudson, P., Remedios, C., Zordan, R., Thomas, K., Clifton, D., Crewden, M., ... Clarke, D. (2010). Clinical practice guidelines for the psychosocial and bereavement support of family caregivers of palliative care patients. Melbourne, Australia: Centre for Palliative Care, St Vincent's Hospital Melbourne.

Jansma, F. F. I., Schure, L. M., \& deJong, B. M. (2005). Support requirements for caregivers of patients with palliative cancer. Patient Education and Counseling, 58, 182-186.

Lobb, E. A., Kristjanson, L. J., Aoun, S. M., Monterosso, L., Halkett, G. K. B., \& Davies, A. (2010). Predictors of complicated grief: A systematic review of empirical studies. Death Studies, 34, 673-698.

Maercker, A., Brewin, C. R., Bryant, R. A., Cloitre, M., Reed, G. M., van Ommeren, M., . ....,Saxena, S. (in press). Proposals for mental disorders specifically associated with stress in the ICD-11. Lancet.

Mather, M. A., Good, P. D., Cavenagh, J. D., \& Ravenscroft, P. J. (2008). Survey of bereavement support provided by Australian palliative care services. Medical Journal of Australia, 188(4), 228-230.

Matsushima, T., Akabayashi, A., \& Nishitateno, K. (2002). The current status of bereavement follow-up in hospice and palliative care in Japan. Palliative Medicine, $16,151-158$.

National Hospice and Palliative Care Organization. (2008). Guidelines for bereavement care in hospice $\left(2^{\text {nd }}\right.$ ed.). Alexandria, VA: Author. 
National Institute for Health and Clinical Excellence. (2004). Guidance on cancer services: Improving supportive and palliative care for adults with cancer. The Manual. London: Author. Accessed $25^{\text {th }}$ January 2011 from: http://guidance.nice.org.uk/CSGSP/Guidance/pdf/English

Neimeyer, R. A. (2008). Grief and bereavement counseling. Accessed $26^{\text {th }}$ July 2011 from http://web.mac.com/neimeyer/Home/Scholarship.html

Neimeyer, R. A., Harris, D. L., Winokuer, H. R., \& Thornton, G. F. (Eds.) (2011). Grief and bereavement in contemporary society: Bridging research and practice. New York: Routledge.

O’Connor, M., Abbott, J.-A., Payne, S., \& Demmer, C. (2009). A comparison of bereavement services provided in hospice and palliative care settings in Australia, the UK and the USA. Progress in Palliative Care, 17, 69-74.

O’Connor, M., \& Lee-Steere, R. (2006). GPs' attitudes to palliative care: A Western Australian rural perspective. Journal of Palliative Medicine, 9(6), 1271-1281.

O’Connor, M., Pugh, J., Jiwa, M., Hughes, J., \& Fisher, C. (2011). The palliative care interdisciplinary team: Where is the community pharmacist? Journal of Palliative Medicine, 14(1), 7-11.

O’Connor, M., Fisher, C., French, L., Halkett, G., Jiwa, M., \& Hughes, J. (2011). Exploring the community pharmacist's role in palliative care: Focusing on the person not just the prescription. Patient Education and Counseling, 83, 458-464.

Palliative Care Australia. (2005). Standards for providing quality palliative care for all Australians ( $4^{\text {th }}$ ed.). Canberra, Australian Capital Territory, Australia: Author. Accessed $22^{\text {nd }}$ March 2011 from http://www.palliativecare.org.au/Portals/46/Standards\%20for\%20providing\%20quali ty\%20palliative\%20care\%20for\%20all\%20Australians.pdf 
Palliative Care Australia. (2010). National Standards Assessment Program: National quality report. Deakin West, Australian Capital Territory, Australia: Author. Accessed $21^{\text {st }}$ September 2011 from http://www.standards.palliativecare.org.au/Portals/20/NSAP\%20National\%20Qualit y\%20Report.pdf

Prigerson, H. G., Silverman, G. K., Jacobs, S.C., Maciejewski, P. K., Kasl, S. V., \& Rosenheck, R. A. (2001). Traumatic grief, disability, and the underutilization of health services: A preliminary examination. Primary Psychiatry, 8, 61-66.

Prigerson, H. G., Vanderwerker, L. C., \& Maciejewski, P. K. (2008). A case for inclusion of prolonged grief disorder in DSM-IV. In M. S. Stroebe, R. O. Hansson, H. Schut, \& W. Stroebe, (Eds.), Handbook of bereavement research and practice: Advances in theory and intervention (pp. 165-186). Washington, DC: American Psychological Association.

Reid, D., Field, D., Payne, S., \& Relf, M. (2006a). Adult bereavement in five English hospices: Participants, organisations and pre-bereavement support. International Journal of Pallative Nursing, 12, 320-327.

Reid, D., Field, D., Payne, S., \& Relf, M. (2006b). Adult bereavement in five English hospices: Types of support. International Journal of Pallative Nursing, 12, 430-437.

Sakaguchi, Y., Tsuneto, S., Takayama, K., Tamura, K., Ikenaga, M., Kashiwagi, T. (2004). Tasks perceived as necessary for hospice and palliative care unit bereavement services in Japan. Journal of Palliative Care, 20, 320-323.

Salau, S., Rumbold, B., \& Young, B. (2007). From concept to care: Enabling community care through a health promoting palliative care approach. Contemporary Nurse, 27, $132-140$. 
Schut, H., \& Stroebe, M. (2005). Interventions to enhance adaptation to bereavement. Journal of Palliative Medicine, 8, S140-146.

Schut, H., Stroebe, M. S., van den Bout, J., \& Terheggen, M. (2001). The efficacy of bereavement interventions: Determining who benefits. In M. S. Stroebe, R. O. Hansson, W. Stroebe, \& H. Schut (Eds.), Handbook of bereavement research: Consequences, coping, and care (pp. 705-727). Washington, DC: American Psychological Association.

Sepúlveda, C., Marlin, A., Yoshida, T., \& Ullrich, A. (2002). Palliative care: The World Health Organization's global perspective. Journal of Pain and Symptom Management, 24, 91-96.

Small, N., \& Hockey, J. (2001). Discourse into practice: The production of bereavement care. In J. Hockey, J. Katz, \& N. Small (Eds.), Grief, mourning and death ritual (pp. 97-124). Buckingham, UK: Open University Press.

Vanderwerker, L. C., Laff, R. E., Kadan-Lottick, N. S., McColl, S., \& Prigerson, H. G. (2005). Psychiatric disorders and mental health service use among caregivers of advanced cancer patients. Journal of Clinical Oncology, 23, 6899-6907.

Victoria Hospice. (2010). Annual report 2009/2010. Victoria, British Columbia, Canada: Author. Accessed $19^{\text {th }}$ July 2011 from http://www.victoriahospice.org/about/annualreports

World Health Organization. (2007). WHO guide for effective programmes: Palliative care. Author. Accessed February 7, 2011, from http://www.who.int/cancer/media/FINAL-Palliative\%20Care\%20Module.pdf 